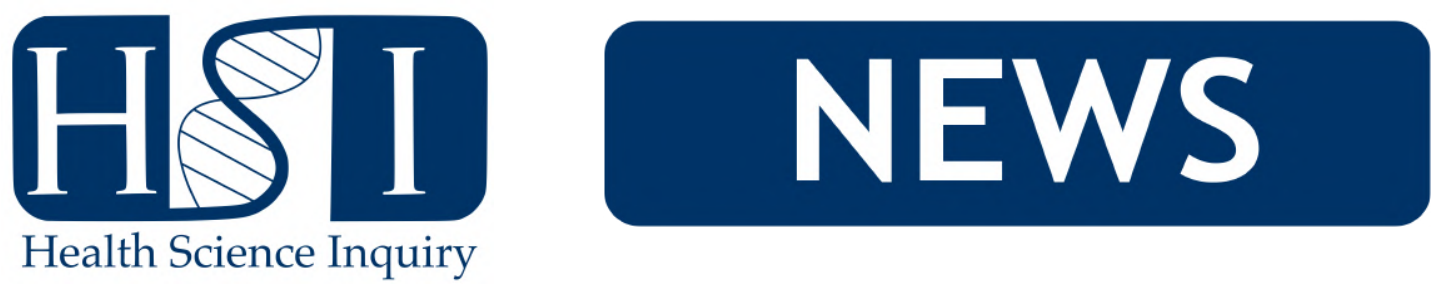

\title{
Climate Change and the Future of Public Health
}

\section{Alanna Miller}

Popular media is saturated with terrifying claims of the effects of global climate change. Our minds absorb the various statistics on the melting of the polar ice caps and rising sea level, the destruction of rainforests and extinction of exotic species, and the pollution of the air and water that the world relies on. Of course, the effects of climate change exist far beyond environmental degradation, and the discussion surrounding climate change cannot be possible without considering the broader implications for public health, which are often ignored in popular media. In accordance with the United Nations (UN), the Intergovernmental Panel on Climate Change (IPCC) released a report in 2018 describing the "impacts of global warming of $1.5^{\circ} \mathrm{C}$ above pre-industrial levels" expected to occur between the years 2030 and 2052 [1]. This increase in global temperature will likely have negative repercussions for public health, including exacerbations of chronic diseases and mental illness, food shortages and malnutrition, human displacement, and increased transmission and susceptibility to infectious diseases $[1,2,3]$.

Extreme weather events, including heat waves, droughts, and floods, are potentially disastrous consequences of climate change. The occurrence of extreme temperatures has been increasing since the 1990s [2], and the IPCC predicts that throughout the $21^{\text {st }}$ century, global temperatures will continue to rise, with more locations experiencing hotter temperatures and fewer cold spells [1]. Extreme heat poses a serious public health threat; it can cause heat stroke and worsen the symptoms of chronic diseases, including cardiovascular disease and chronic respiratory disease [4]. In Quebec and Ontario, 2018 was host to one of the hottest summers yet, and a heatwave in July was ultimately responsible for claiming the lives of over 80 individuals in Quebec alone [5]. Those over the age of 65, people who engage in vigorous outdoor manual labour, and individuals residing in urban areas are at the greatest risk of heat-related morbidity and mortality $[2,4]$.

Extreme weather events can also indirectly impact human health by affecting food supply and availability. Agricultural yields are disturbed by flooding and drought events, which may lead to food shortages and malnutrition in severe circumstances [2]. These shortfalls in agricultural production are more likely to affect those living in poverty in middleand low-income countries $[1,2]$. In addition to food shortages, extreme weather events can cause perilous damage to the built environment, destroy homes and infrastructure, and can result in the displacement of people and severe economic loss [2]. Again, these effects are more detrimental to individuals in low- and middle-income countries, and can negatively impact the mental health of those affected [2].

The most researched topic regarding the implications of climate change on public health is the transmission of infectious diseases carried by insects. Increased transmission of malaria and dengue, known as the 'Big Two', is expected to coincide with the warming of temperatures and increased regional precipitation associated with climate change [1,3]. Both malaria and dengue are carried by mosquitos and are common to parts of South and Central America, Asia and Africa. However, considering the future implications of climate change, we must pose the question: Could malaria and dengue spread to more temperate regions, such as those here in Canada?

Dr. Mark Loeb of McMaster University is a professor for the Department of Pathology and Molecular Medicine, and studies infectious diseases spread by insects, including West Nile and dengue. He believes that "in the long-term it is possible for such diseases to end up in Canada". Aside from malaria and dengue, there are many other diseases, including Lyme disease, an infection transmitted by ticks, which is currently present in certain regions of Canada. Lyme disease can become a chronic infection characterized by severe headaches, joint and muscle pain, arthritis, heart disorder and neurological disorder, and can be very debilitating to those infected. Dr. Loeb said there has already been an observed increase in the geographical spread of the tick-borne infection and suggests that in order to protect against the increased spread of infectious diseases, we must focus on reducing the trends in climate change and the development of effective and accessible vaccines.

There is no doubt that climate change will continue to have an impact on public health, and this will become even more detrimental if public health systems are not prepared to cope with these changes. In Ottawa, ON, on February 5, 2019, the Canadian Public Health Association (CPHA) released a statement urging federal political parties to acknowledge the severity of climate change and its negative impact on public health [6]. The CPHA challenged the federal government to develop a plan for Canada to reduce carbon emissions while prioritizing health, create policies and programs for 
corporations and communities to transition to a low carbon economy, and provide funding and support to ensure public health units and communities are able to respond to the mental and physical health needs of Canadians affected by climate change [6]. When analyzing the amount of funding countries allocated to public health adaptation, the greatest increase in funding was observed in lower- and middle-income countries [2]. Considering that the brunt of climate change will be felt in low- and middle-income countries [2], this increase in funding for public health adaptation is encouraging. Furthermore, the UN suggests that reducing poverty and encouraging socioeconomic development are the best ways to increase resiliency within these countries, but unfortunately, funding available for these initiatives is still insufficient [2].

Since pre-industrial times, human activity has caused an increase in average global temperature by approximately $1.0^{\circ} \mathrm{C}$, and the IPCC suggests with strong evidence that we will observe another $0.5^{\circ} \mathrm{C}$ increase by the year $2052[1]$. Climate change is therefore inevitable, and it is important that we prepare our public health infrastructure now to improve our future resiliency. Climate change will have negative repercussions for public health, including exacerbations of chronic diseases and mental illness, food shortages and malnutrition, human displacement, and increased transmission of infectious diseases $[1,2,3]$. Key ways to adapt public health systems for the future consequences of climate change are to reduce poverty and improve socioeconomic development [2]. On a corporate and individual level, we must also focus on limiting the anthropogenic factors, such as greenhouse gas emissions, to reduce any future negative impacts of climate change on public health. Instead of allowing the effects of climate change to play out with our compliance, it is now that each country, corporation and individual must take action to ensure that we are protecting both our planet and our health.

\section{REFERENCES}

[1] Intergovernmental Panel on Climate Change. Global warming of $1.5^{\circ} \mathrm{C} ; 2018$.

[2] Watts N, Amann M, Arnell N, Ayeb-Karlsson S, Belesova K, Berry H, et al. The 2018 report of the Lancet Countdown on health and climate change: shaping the health of nations for centuries to come. The Lancet. 2018;392(10163):24792514 .

[3] Ogden NH. Climate change and vector-borne diseases of public health significance. FEMS microbiology letters. 2017;364(19):fnx186.

[4] Kjellstrom T, Butler AJ, Lucas RM, Bonita R. Public health impact of global heating due to climate change: potential effects on chronic non-communicable diseases. International journal of public health. 2010;55(2):97-103.

[5] National Post. New research predicts heat waves in Canada could become more frequent and five times more deadly; 2018. Available from: https://nationalpost.com/news/canada/ new-research-predicts-heat-waves-in-canada-could-become-more-frequent-and-five-times-more-deadly.

[6] Canadian Public Health Association. Health professionals to federal political parties: action needed to prevent catastrophic climate change;. Available from: https://www.cpha.ca/ health-professionals-federal-political-parties-action-needed-prevent-catastrophic-climate-change.

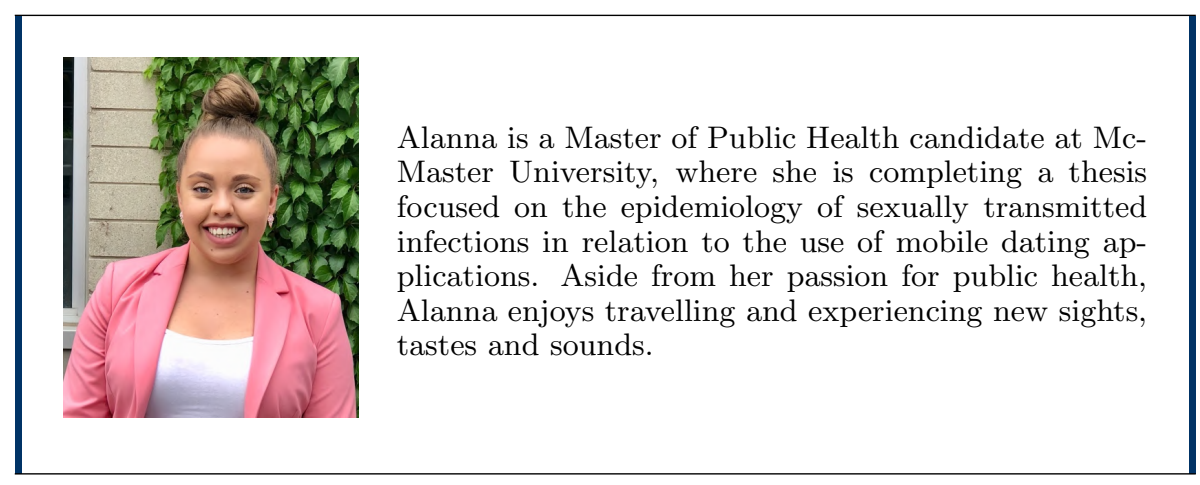

